\title{
Pacific
}

Journal of

Mathematics

\section{UNIVERSAL CLASSES OF ORLICZ FUNCTION SPACES}

Francisco Luis Hernández Rodríguez and Cesar Ruiz 


\title{
UNIVERSAL CLASSES OF ORLICZ FUNCTION SPACES
}

\author{
Francisco L. Hernández and César Ruiz
}

\begin{abstract}
It is shown that for each $0<p<q<\infty$ the space $L^{p}(0, \infty)+$ $L^{q}(0, \infty)$, defined as in Interpoliation Theory, is universal for the class of all Orlicz function spaces $L^{\psi}$ with Boyd indices strictly between $p$ and $q$ (i.e. every Orlicz function space $L^{\psi}$ is orderisomorphically embedded into $\left.L^{p}(0, \infty)+L^{q}(0, \infty)\right)$. The extreme case of spaces having Boyd indices equal to $p$ or $q$ is also studied. In particular every space $L^{r}(0, \infty)+L^{s}(0, \infty)$ embeds isomorphically into the sum $L^{p}(0, \infty)+L^{q}(0, \infty)$ for any $0<p \leq r \leq s<q<\infty$.
\end{abstract}

0. Introduction. It is a well-known fact from Interpolation Theory (cf. [L-T II] Proposition 2.b.3) that given $1 \leq p<q \leq \infty$, every rearrangement invariant (r.i.) Banach function space $X$ on the interval $(0, \infty)$ with Boyd indices between $p$ and $q$, is an intermediate space between the spaces $L^{p}(0, \infty) \cap L^{q}(0, \infty)$ and $L^{p}(0, \infty)+L^{q}(0, \infty)$. This means that $L^{p}(0, \infty) \cap L^{q}(0, \infty) \subset X \subset L^{p}(0, \infty)+L^{q}(0, \infty)$ with continuous inclusions.

One of the purposes of this paper is to study the universality of the spaces $L^{p}(0, \infty)+L^{q}(0, \infty)$ with respect to classes of intermediate r.i. function spaces $X$, in the sense of whether the above inclusion $X \subset L^{p}(0, \infty)+L^{q}(0, \infty)$ can be replaced by suitable isomorphic embeddings of $X$ into $L^{p}(0, \infty)+L^{q}(0, \infty)$. At the same time, these spaces $L^{p}(0, \infty)+L^{q}(0, \infty)$ can be regarded as Orlicz function spaces $L^{\varphi}(0, \infty)$, taking the Orlicz function $\varphi(x)=x^{p} \wedge x^{q}=\min \left(x^{p}, x^{q}\right)$, and, consequently, we are also interested in finding universal Orlicz function spaces for classes of quasi-Banach Orlicz function spaces $L^{\psi}(0, \infty)$.

These questions are motived in part by the existence of positive results of universality in the context of Orlicz sequence spaces: J. Lindenstrauss and L. Tzafriri (cf. [L-T I] Theorem 4.b.12) proved that there exist universal Orlicz sequence spaces $l^{\varphi}$ with arbitrary prefixed indices $1 \leq p<q<\infty$ such that every Orlicz sequence space $l^{\psi}$ with indices between $p$ and $q$ is isomorphic to a (complemented) subspace of $l^{\varphi}$.

We show here that, in general, for $0<p<q<\infty$, the spaces $L^{p}(0, \infty)+L^{q}(0, \infty)$ are universal for the class of all Orlicz function 
spaces $L^{\psi}(0, \infty)$ with Boyd indices strictly between $p$ and $q$. On the other hand, this universality of the spaces $L^{p}(0, \infty)+L^{q}(0, \infty)$ does not hold for every intermediate r.i. function space $X$ : there are Lorentz function spaces which cannot be embedded into $L^{p}(0, \infty)+$ $L^{q}(0, \infty)$.

These results are deduced from the two main theorems presented in $\S 2$ (Theorems 3.A and 3.B), which are obtained through results of representing the Orlicz function $\psi$, between $p$ and $q$, in an integral form with respect to the function $x^{p} \wedge x^{q}=\varphi(x)$, i.e. there exists a probability measure $\nu$ on $(0, \infty)$ such that, up to equivalence,

$$
\psi(x)=\int_{0}^{\infty} \frac{\varphi(x s)}{\varphi(s)} d \nu(s) .
$$

Several remarkable consequences are given in $\S 3$. Thus, in Proposition 6 we obtain sufficient conditions on the embedding of Orlicz function spaces $L^{\psi}(0, \infty)$ into Orlicz spaces over finite measure $L^{\varphi}[0,1]$, which were obtained by Bretagnolle and Dacunha-Castelle ([B-DC]) by using probabilistic tools (see also [J-M-S-T]).

Corollary 8 extends a recent result of S. J. Dilworth ([D]) about the scale of spaces $L^{2}(0, \infty)+L^{q}(0, \infty)$ for $2<q<\infty$. We show that for any $0<p \leq r \leq s<q<\infty$ the spaces $L^{r}(0, \infty)+L^{s}(0, \infty)$ are order-isomorphic to sublattices of the space $L^{p}(0, \infty)+L^{q}(0, \infty)$.

We would like to thank Ives Raynaud for helpful suggestions on this topic.

1. Preliminaries. Let us start with some notations and definitions. By $\varphi$ we denote an Orlicz function, i.e. a non-decreasing continuous function defined for $x \geq 0$ so that $\varphi(0)=0$ and $\varphi(1)=1$. Two Orlicz functions $\varphi$ and $\psi$ are equivalent at $\infty$, denoted by $\psi \stackrel{\infty}{\sim} \psi$ (resp. at $0, \varphi \stackrel{0}{\sim} \psi$ ) if there exist a constant $C \geq 1$ and $x_{0}>0$ such that $C^{-1} \varphi(x) \leq \psi(x) \leq C \varphi(x)$ for every $x \geq x_{0}$ (resp. $\left.0<x \leq x_{0}\right)$. When $\varphi$ and $\psi$ are equivalent at $\infty$ and at 0 we say that $\varphi$ and $\psi$ are equivalent, i.e. $\varphi \sim \psi$. Given $0<p<\infty$, an Orlicz function $\varphi$ is $p$-convex (resp. $p$-concave) if $\varphi\left(x^{1 / p}\right)$ is a convex function of $x$ (resp. a concave function).

$(I, \mu)$ means the Lebesgue measure space for $I=[0,1]$ or $(0, \infty)$. The Orlicz function space $L^{\varphi}(I)$ is defined as the set of equivalence classes of $\mu$-measurable scalar functions on $I$ such that

$$
m(f / u)=\int_{I} \varphi(|f(t)| / u) d \mu(t)<\infty \quad \text { for some } u>0 .
$$


The space $L^{\varphi}(I)$ is an $F$-space when we consider the $F$-norm

$$
|f|_{\varphi}=\inf \{u>0: m(f / u) \leq u\} .
$$

It is well-known ([M-O]) that an Orlicz space $L^{\varphi}(0, \infty)$ is $p$-normable $(0<p \leq 1)$ if and only if there exists a $p$-convex function $\psi$ such that $L^{\varphi}(0, \infty)=L^{\psi}(0, \infty)$, where $L^{\psi}(0, \infty)$ is endowed with the $p$-norm,

$$
\left.\|f\|_{\psi}=\inf \left\{u>0: \int_{0}^{\infty} \psi(\mid f(t)) \mid / u^{1 / p}\right) d \mu(t) \leq 1\right\},
$$

so $\left(\|f\|_{\psi}\right)^{1 / p}$ is a quasi-norm (in the case $p=1,\|\|_{\psi}$ is the usual Luxemburg norm). Moreover, recall that if two Orlicz functions $\varphi$ and $\psi$ are equivalent (resp. $\varphi \stackrel{\infty}{\sim} \psi$ ), then $L^{\varphi}(0, \infty)=L^{\psi}(0, \infty)$ (resp. $L^{\varphi}[0,1]=L^{\psi}[0,1]$ ) and the identity map is an isomorphism.

An Orlicz function $\varphi$ satisfies the $\Delta_{2}$-condition (resp. at $\infty$ ) if there exists a constant $M>0$ such that $\varphi(2 x) \leq M \varphi(x)$ for $x>0$ (resp. $x>x_{0}$ for some $\left.x_{0}>0\right) . L^{\varphi}(0, \infty)$ is separable iff $\varphi$ verifies the $\Delta_{2}$-condition.

For basic properties of Orlicz spaces we refer to [Lu] and [Mu]. For Banach structure properties see [L-T II], [J-M-S-T], [H-Ro] and [R].

Following the terminology of Woo ([W]) we have the following:

Definition 1. Let $0<p<q<\infty$. An Orlicz function $\varphi$ is said to be between $p$ and $q$ if $\varphi(x) / x^{p}$ is non-decreasing on $\mathbb{R}^{+}$and $\varphi(x) / x^{q}$ is non-increasing on $\mathbb{R}^{+}$. By $\mathscr{K}(p, q)$ is denoted the set of all Orlicz functions $\varphi$ between $p$ and $q$.

It is clear that every Orlicz function $\varphi$ which is $p$-convex and $q$ concave belongs to $\mathscr{K}(p, q)$. If $p \leq p_{1}$ and $q_{1} \leq q$, then $\mathscr{K}\left(p_{1}, q_{1}\right)$ $\subset \mathscr{K}(p, q)$. Also it is very easy to show that a function $\varphi$ with continuous derivative belongs to $\mathscr{K}(p, q)$ iff

$$
p \leq x \varphi^{\prime}(x) / \varphi(x) \leq q \text { for every } x>0 .
$$

Proposition 2 ([M] and [W]). Let $0<p<q<\infty$. For every Orlicz function $\varphi \in \mathscr{K}(p, q)$ there exists a p-convex and $q$-concave Orlicz function $\psi$ with continuous second derivative, such that $\varphi$ and $\psi$ are equivalent.

The following result of universality for Orlicz sequence spaces is given in [L-T I], Theorem 4.b.12: Given $1 \leq p<q<\infty$ there exists an Orlicz function $\varphi_{p, q}=\varphi$ which is $p$-convex and $q$-concave at 0 , such that for every Orlicz function $\psi p$-convex and $q$-concave 
at 0 , the Orlicz sequence space $l^{\psi}$ embeds isomorphically into $l^{\varphi}$. Moreover $l^{\varphi}$ has a 1-complemented copy of $l^{\psi}$.

We analyze here possible results of universality like the above one in the context of Orlicz function spaces $L^{\varphi}(0, \infty)$. In [J-M-S-T], Theorem 7.1, it is proved that if a rearrangement invariant function space $X([0,1])$, in particular an Orlicz function space $L^{\psi}[0,1]$, is isomorphic to a complemented subspace of a reflexive Orlicz function space $L^{\varphi}(0, \infty)$, then $L^{\psi}[0,1]=L^{\varphi}[0,1]$ or $L^{\psi}[0,1]=L^{2}[0,1]$, up to an equivalent renorming. It follows from this result that there are no Orlicz function spaces $L^{\varphi}(0, \infty)$ which are complementably universal for classes of function spaces $L^{\psi}(0, \infty)$. This leads us to study the existence of universal spaces $L^{\varphi}(0, \infty)$ just for (non-complemented) isomorphic embedding.

We will make use of the following remarkable result given in $[\mathbf{J}-$ M-S-T]: Every reflexive Orlicz function space $L^{\varphi}[0,1]$ can be represented isomorphically as the Orlicz space $L^{\bar{\varphi}}(0, \infty)$, where $\bar{\varphi}$ is the function defined by $x^{2}$ at 0 and by $\varphi(x)$ at $\infty$.

2. Main results. Given $0<p<q<\infty$, let us denote by $x^{p} \wedge x^{q}$ the Orlicz function defined by $\min \left\{x^{p}, x^{q}\right\}=\varphi(x)$ for every $x \geq 0$. Obviously the function $x^{p} \wedge x^{q}$ belongs to the class $\mathscr{K}(p, q)$.

The main results of this paper are the following:

Theorem 3.A. Let $0<p<q<\infty$. For every Orlicz function $\psi \in \mathscr{K}(p, q)$, which is non-equivalent to the function $x^{p}$ and $x^{q}$ neither at 0 nor at $\infty$, the Orlicz space $L^{\psi}(0, \infty)$ is order-isomorphic to a sublattice of $L^{x^{p} \wedge x^{q}}(0, \infty)$.

TheOREM 3.B. Let $0<p<q<\infty$. For every Orlicz function $\psi \in \mathscr{K}(p, q)$ such that $\psi(x)=x^{p}$ at $\infty$ and $\psi$ is non-equivalent to the function $x^{p}$ and $x^{q}$ at 0 , the Orlicz space $L^{\psi}(0, \infty)$ is orderisomorphic to a sublattice of $L^{x^{p} \wedge x^{q}}(0, \infty)$.

In order to prove these theorems we give some preliminary results. Given a positive function $g$ of an Orlicz space $L^{\varphi}(0, \infty)$ with $\|g\|_{\varphi}=1$, we consider the Orlicz function $\Phi_{g}$ defined by

$$
\Phi_{g}(x)=\int_{0}^{\infty} \varphi(x g(s)) d s \quad \text { for } x \geq 0 .
$$

It holds that the Orlicz space $L^{\Phi_{g}}(0, \infty)$ is order-isomorphic (and isometric) to a sublattice of $L^{\varphi}(0, \infty)$. This follows from the fact that the map $T: L^{\Phi_{g}}(0, \infty) \rightarrow L^{\varphi}((0, \infty) \times(0, \infty))$ defined by $T(f)=$ 
$f \otimes g$, where $f \otimes g(t, s)=f(t) g(s)$, is an (isometric) order-isomorphism into $L^{\varphi}((0, \infty) \times(0, \infty))$ (see [J-M-S-T] pp. 189 for the proof in the case of convex functions $\varphi$, which also works in the $p$-convex case).

Proposition 4. Let $\varphi$ be an Orlicz function verifying the $\Delta_{2}$-condition and $\nu$ be a probability measure on $(0, \infty)$. If $\psi$ is the Orlicz function defined by

$$
\psi(x)=\int_{0}^{\infty} \varphi(x s) / \varphi(s) d \nu(s) \quad \text { for } x \geq 0
$$

then the Orlicz space $L^{\psi}(0, \infty)$ is order-isometric to a sublattice of $L^{\varphi}(0, \infty)$.

Proof. It is similar to the one given in [J-M-S-T], Theorem 7.7, in the case of normed Orlicz spaces over the $[0,1]$ interval. Let us sketch it for the sake of completeness.

Let us define a positive function $g$ by its distribution function:

$$
\mu\{s: g(s) \in A\}=\int_{A} 1 / \varphi(s) d \nu(s)
$$

for every measurable set $A$ in $(0, \infty)$. For each positive $\mu$-measurable function $h$ on $(0, \infty)$

$$
\int_{0}^{\infty} h(s) / \varphi(s) d \nu(s)=\int_{0}^{\infty} h(g(t)) d \mu(t) .
$$

In particular for $h=\varphi$, we get $\int_{0}^{\infty} \varphi(g(t)) d \mu(t)=1$, so $g \in$ $L^{\varphi}(0, \infty)$ and $\|g\|_{\varphi}=1$. Now, taking $h(s)=\varphi(x s)$ for $x \geq 0$, we obtain that $\psi(x)=\Phi_{g}(x)$. Hence we conclude that

$$
L^{\psi}(0, \infty)=L^{\Phi_{g}}(0, \infty) \cong L^{\varphi}(0, \infty) .
$$

The next results allow us to represent Orlicz functions of the class $\mathscr{K}(p, q)$ in an intergral form of type (1):

Proposition 5.A. Given $0<p<q<\infty$ and the Orlicz function $\varphi(x)=x^{p} \wedge x^{q}$. Every Orlicz function $\psi \in \mathscr{K}(p, q)$, which is nonequivalent to the functions $x^{p}$ and $x^{q}$ neither at 0 nor at $\infty$, can be represented, up to equivalence, as

$$
\psi(x)=\int_{0}^{\infty} \varphi(x s) / \varphi(s) d \nu(s)
$$

for some probability measure $\nu$ on $(0, \infty)$.

Proposition 5.B. Given $0<p<q<\infty$ and $\varphi(x)=x^{p} \wedge x^{q}$. Every Orlicz function $\psi \in \mathscr{K}(p, q)$ such that $\psi(x)=x^{p}$ at $\infty$, 
and which is non-equivalent to the function $x^{p}$ and $x^{q}$ at 0 , can be represented, up to equivalence, as

$$
\psi(x)=\int_{0}^{\infty} \varphi(x s) / \varphi(s) d \nu(s)
$$

for some probability measure $\nu$ on $(0, \infty)$.

Proof (5.A). We will take some ideas of the proof of Theorem 4.5 in [W]. We assume by Proposition 2 that $\psi$ is a continuous second derivative. Consider the function

$$
N(x)=\frac{\psi\left(x^{1 / q-p}\right)}{x^{p / q-p}} .
$$

It follows easily from the properties of the function $\psi$ that $N$ is a non-decreasing function, $N(0)=0$, and satisfies

$$
\lim _{x \rightarrow 0} x N^{\prime}(x)=0 \quad \text { and } \quad \lim _{x \rightarrow \infty} N^{\prime}(x)=0 .
$$

We define the function

$$
\begin{aligned}
\Phi(x)= & \int_{0}^{\infty} t^{p-2 q-1} N^{\prime \prime}\left(t^{p-q}\right)(p-q) \varphi(x t) d t \\
= & x^{q} \int_{0}^{1 / x} t^{p-2 q-1} N^{\prime \prime}\left(t^{p-q}\right)(p-q) t^{q} d t \\
& +x^{p} \int_{1 / x}^{\infty} t^{p-2 q-1} N^{\prime \prime}\left(t^{p-q}\right)(p-q) t^{p} d t
\end{aligned}
$$

Making the variable change $u=t^{p-q}$,

$$
\begin{aligned}
\Phi(x) & =x^{q} \int_{\infty}^{(1 / x)^{p-q}} N^{\prime \prime}(u) d u+x^{p} \int_{(1 / x)^{p-q}}^{0} u N^{\prime \prime}(u) d u \\
& =x^{q} N\left((1 / x)^{p-q}\right)=\psi(x) .
\end{aligned}
$$

Now, denoting by $\widetilde{N}$ the function

$$
\tilde{N}(t)=t^{2 p-2 q-1} N^{\prime \prime}\left(t^{p-q}\right)(p-q),
$$

we have the expressions

$$
1=\psi(1)=\int_{0}^{1} t^{q-p} \tilde{N}(t) d t+\int_{1}^{\infty} \tilde{N}(t) d t=\int_{0}^{\infty} \frac{t^{p} \wedge t^{q}}{t^{p}} \tilde{N}(t) d t
$$

and in general for $x>0$

$$
\begin{aligned}
\psi(x) & =x^{q} \int_{0}^{1 / x} t^{q} \frac{\tilde{N}(t)}{t^{p}} d t+x^{p} \int_{1 / x}^{\infty} t^{p} \frac{\tilde{N}(t)}{t^{p}} d t \\
& =\int_{0}^{\infty} \frac{(x t)^{q} \wedge(x t)^{p}}{t^{q} \wedge t^{p}} \frac{t^{q} \wedge t^{p}}{t^{p}} \tilde{N}(t) d t
\end{aligned}
$$


Hence, taking the probability measure $\nu$ on $(0, \infty)$ defined by

$$
\nu(A)=\int_{A} \frac{t^{q} \wedge t^{p}}{t^{p}} \tilde{N}(t) d t
$$

we are done.

Proof (5.B). We will keep the above notation. So, reasoning as before, we consider the function

$$
\Phi(x)=\frac{1}{K} \int_{1}^{\infty}(p-q) t^{p-2 q-1} N^{\prime \prime}\left(t^{p-q}\right)(x t)^{p} \wedge(x t)^{q} d t
$$

for every $x \geq 0$, where $N$ is defined as in the above proof, but only on the interval $[0,1]$. And the constant $K$ is

$$
K=\int_{1}^{\infty}(p-q) t^{p-2 q-1} N^{\prime \prime}\left(t^{p-q}\right) t^{p} d t
$$

By integrating, we get

$$
\Phi(x)=1 / K\left(x^{p} N\left((1 / x)^{p-q}\right)-x^{q} N^{\prime}(1)\right) .
$$

Now, from the definition of $N$, it follows that there exists $0<x_{0} \leq 1$ such that

$$
\frac{x^{p} N\left((1 / x)^{p-q}\right)-x^{q} N^{\prime}(1)}{\psi(x)}=1-N^{\prime}(1) \frac{x^{q}}{\psi(x)} \geq 1 / 2
$$

for every $x \in\left[0, x_{0}\right]$. Therefore $\psi \stackrel{0}{\sim} \Phi$.

Proceeding as in 5.A we consider the function

$$
\tilde{N}(t)=\frac{1}{K} t^{2 p-2 q-1} N^{\prime \prime}\left(t^{p-q}\right)(p-q)
$$

obtaining that

$$
\psi(x) \sim \int_{0}^{\infty} \frac{(x t)^{p} \wedge(x t)^{q}}{t^{p} \wedge t^{q}} d \nu(t)
$$

where $\nu$ is a probability measure on $(0, \infty)$ defined by $\nu([0,1])=0$ and for every measurable set $A$ in $(1, \infty)$

$$
\nu(A)=\int_{A} \frac{t^{q} \wedge t^{p}}{t^{p}} \tilde{N}(t) d t
$$

Proof of Theorems 3.A and 3.B. It follows directly from Propositions 5.A and 5.B together with Proposition 4.

3. Consequences. We start this section giving an embedding result for Orlicz spaces over finite measure space. 
Proposition 6. Let $\varphi$ be a convex Orlicz function at $\infty$ which is $q$ concave at $\infty$ for some $q<2$. Then the Orlicz function space $L^{\varphi}[0,1]$ is universal for the class of all Orlicz function spaces $L^{\psi}(0, \infty)$ with $\psi \in \mathscr{K}(r, s)$ and $q<r \leq s<2$.

Proof. From $\varphi \in \mathscr{K}(1, q)$, it follows that $\varphi(x) / x^{r}$ is a decreasing function for $x \geq 1$. So

$$
\int_{0}^{1} \varphi\left((1 / t)^{1 / r}\right) d t \leq \int_{0}^{1}(1 / t)^{s / r} d t<\infty
$$

and, by Proposition 8.9 in [J-M-S-T] (see also [L-T II] Theorem 2.f.4) we obtain that the space $L^{r}[0,1]$ is isometrically isomorphic to a subspace of $L^{\varphi}[0,1]$. Now, using the important isomorphic representation of $L^{r}[0,1]$ as the space $L^{x^{r} \wedge x^{2}}(0, \infty)$ ([J-M-S-T] Theorem 8.6) together with Theorem 3.A we conclude that

$$
L^{\psi}(0, \infty) \subsetneq L^{r}[0,1] \subsetneq L^{\varphi}[0,1]
$$

REMARK. The above result was stated implicitly by Bretagnolle and Dacunha-Castelle in [B-DC].

Notice that Proposition 6 extends a well-known result of Bretagnolle, Dacunha-Castelle and Krivine ([B-DC-K]), obtained by using $p$-stable random variables: If $1 \leq p \leq r<2$, then the space $L^{r}[0,1]$ is (isometrically) isomorphic to a subspace of $L^{p}[0,1]$.

Let us remark that here we have not used probability tools in the proof of Theorems 3.A and 3.B, but when we prove Proposition 6 we do implicitly because we make use of the Theorem 8.6 of [J-M-S-T] which requires Poisson processes. (Also notice that is the reason of losing the isometric property.)

We turn now to give a representation of the universal Orlicz spaces in Theorems 3.A and 3.B as a sum $L^{p}(0, \infty)+L^{q}(0, \infty)$, which are well-known spaces in Interpolation Theory (see [L-T II]). As a consequence we will extend a recent result of S. Dilworth ([D]) on the scale of spaces $L^{2}(0, \infty)+L^{q}(0, \infty)$.

Given $L^{\varphi}(0, \infty)$ and $L^{\psi}(0, \infty)$ the space sum $L^{\varphi}(0, \infty)+$ $L^{\psi}(0, \infty)$ is the space of all functions $f$ on $(0, \infty)$ which can be written as $g+h$ such that $g \in L^{\varphi}(0, \infty)$ and $h \in L^{\psi}(0, \infty)$, endowed with the norm (or quasi-norm) defined by

$$
\|f\|_{\varphi+\psi}=\inf \left\{\|g\|_{\varphi}+\|h\|_{\psi}: f=g+h\right\} .
$$


Proposition 7. If $\varphi$ and $\psi$ are Orlicz functions verifying the $\Delta_{2}$ condition, $\varphi(x) \leq \psi(x)$ for $0 \leq x \leq 1$, and $\psi(x) \leq \varphi(x)$ for $x \geq$ 1 , then $L^{\varphi}(0, \infty)+L^{\psi}(0, \infty)=L^{\varphi \wedge \psi}(0, \infty)$, the identity being an isomorphism.

Proof. Given $f \in L^{\varphi \wedge \psi}(0, \infty)$, we consider the functions $f^{1}=$ $f \chi_{A_{1}}$ and $f^{2}=f \chi_{A_{2}}$ where $A_{1}=|f|^{-1}([0,1])$ and $A_{2}=|f|^{-1}(1, \infty)$. Hence $f^{1} \in L^{\varphi}(0, \infty)$ and $f^{2} \in L^{\psi}(0, \infty)$, so the inclusion $L^{\varphi \wedge \psi}(0, \infty)$ $\subset L^{\varphi}(0, \infty)+L^{\psi}(0, \infty)$ is continuous. Indeed, if

$$
\left\|f_{n}\right\|_{\varphi \wedge \psi} \underset{n \rightarrow \infty}{\longrightarrow} 0
$$

then

$$
\left\|f_{n}^{1}\right\|_{\varphi} \underset{n \rightarrow \infty}{\longrightarrow} 0 \quad \text { and } \quad\left\|f_{n}^{2}\right\|_{\psi} \underset{n \rightarrow \infty}{\longrightarrow} 0
$$

(cf. [Mu], Theorem 1.6). Hence,

$$
\left\|f_{n}\right\|_{\varphi+\psi} \underset{n \rightarrow \infty}{\longrightarrow} 0 \text {. }
$$

Now, we show that the above inclusion is also onto, which finishes the proof by the Open Mapping Theorem.

If $f \in L^{\varphi}(0, \infty)+L^{\psi}(0, \infty)$ with $f=g+h, g \in L^{\varphi}(0, \infty)$ and $h \in L^{\psi}(0, \infty)$, consider $g^{1}, g^{2}, h^{1}$, and $h^{2}$ as before. Then $g^{2} \in L^{\psi}(0, \infty), h^{1} \in L^{\varphi}(0, \infty)$, and $f=g^{1}+g^{2}+h^{1}+h^{2}$. It is clear that $g^{1}+h^{1} \in L^{\varphi \wedge \psi}(0, \infty)$, since $\frac{\left|g^{1}(t)+h^{1}(t)\right|}{2} \leq 1$ for every $t \geq 0$. Let us see that $g^{2}+h^{2} \in L^{\varphi \wedge \psi}(0, \infty)$. If $A=\left|g^{2}+h^{2}\right|^{-1}([0,1])$, we have $\left|g^{2}+h^{2}\right| \chi_{A} \leq\left|g^{2}\right| \chi_{A}$; hence

$$
\int_{A} \varphi\left(\left|g^{2}+h^{2}\right|\right) d t \leq \int_{A} \varphi\left(\left|g^{2}\right|\right) d t<\infty
$$

so $\left(g^{2}+h^{2}\right) \chi_{A} \in L^{\varphi \wedge \psi}(0, \infty)$.

Finally, as $g^{2}+h^{2} \in L^{\psi}(0, \infty)$, we have

$$
\left(g^{2}+h^{2}\right) \chi_{(0, \infty) \backslash A} \in L^{\varphi \wedge \psi}(0, \infty),
$$

and we can conclude that $f \in L^{\varphi \wedge \psi}(0, \infty)$.

A direct consequence of the above proposition and Theorems 3.A and 3.B is the following:

Corollary 8. Let $0<p \leq r \leq s<q$. The space $L^{r}(0, \infty)+$ $L^{s}(0, \infty)$ is order-isomorphic to a sublattice of $L^{p}(0, \infty)+L^{q}(0, \infty)$.

The case $p=r=s$ follows from the fact

$$
L^{p}(0, \infty) \approx L^{p}[0,1] \subsetneq L^{x^{p} \wedge x^{q}}(0, \infty) .
$$


REMARK. The above result in the particular case of $p=r=2$ has been obtained by S. Dilworth in [D], Theorem 5.7, using a different argument, and giving several structure properties of the scale of spaces $L^{2}(0, \infty)+L^{q}(0, \infty)$ for $2<q<\infty$.

We pass now to give more consequences of Theorems 3.A and 3.B, related with the Boyd indices of rearrangement invariant (r.i.) function spaces. Let us recall their definition, following [L-T II], where they are given as the converses of the original Boyd indices in [Bo].

Let $X$ be a r.i. Banach function space. For $0<s<\infty$, we define the dilation operators $D_{s}$ on $X$ by $\left(D_{s} f\right)(t)=f(t / s)$ for $0 \leq t<\infty$, which are linear and continuous. Now, the Boyd indices $p_{X}$ and $q_{X}$ are defined by

$$
p_{X}=\lim _{s \rightarrow \infty} \frac{\log (s)}{\log \left(\left\|D_{s}\right\|_{X}\right)}=\sup _{s>1} \frac{\log (s)}{\log \left(\left\|D_{s}\right\|_{X}\right)}
$$

and

$$
q_{X}=\lim _{s \rightarrow 0^{+}} \frac{\log (s)}{\log \left(\left\|D_{s}\right\|_{X}\right)}=\inf _{0<s<1} \frac{\log (s)}{\log \left(\left\|D_{s}\right\|_{X}\right)} .
$$

It holds that $1 \leq p_{X} \leq q_{X} \leq \infty$.

In Interpolation Theory the following result is known (see [L-T II], Proposition 2.b.3), showing the r.i. Banach spaces are intermediate spaces between the spaces $L^{p}(0, \infty) \cap L^{q}(0, \infty)$, with the norm of the maximum, and $L^{p}(0, \infty)+L^{q}(0, \infty)$.

Proposition 9. Let $X$ be an ri. Banach function space on $(0, \infty)$. Then, for every $1<p<p_{X} \leq q_{X}<q \leq \infty$, we have

$$
L^{p}(0, \infty) \cap L^{q}(0, \infty) \subset X \subset L^{p}(0, \infty)+L^{q}(0, \infty)
$$

with the inclusion map being continuous.

Let us show here that when we restrict ourselves to considering Orlicz function spaces $L^{\varphi}(0, \infty)=X$, we can substitute the above inclusion $X \subset L^{p}(0, \infty)+L^{q}(0, \infty)$ by an order-isomorphism.

When a r.i. function space $X$ is an Orlicz function space $L^{\varphi}(0, \infty)$, the associated Boyd indices $p_{X}$ and $q_{X}$ coincide with the Matuszewska-Orlicz indices $\sigma_{\varphi}^{a}$ and $s_{\varphi}^{a}$ respectively (see [Bo], also [Ma]). Let us recall that these indices $\sigma_{\varphi}^{a}$ and $s_{\varphi}^{a}$, related with the growing behavior of the Orlicz function $\varphi$ in the positive real line, are defined by

$$
\sigma_{\varphi}^{a}=\lim _{\lambda \rightarrow \infty} \frac{\log \left(\inf _{u>0}\{\varphi(\lambda u) / \varphi(u)\}\right)}{\log (\lambda)}
$$


and

$$
s_{\varphi}^{a}=\lim _{\lambda \rightarrow \infty} \frac{\log \left(\sup _{u>0}\{\varphi(\lambda u) / \varphi(u)\}\right)}{\log (\lambda)} .
$$

Hence, given $0<p<q<\infty$, for any Orlicz function space $L^{\varphi}(0, \infty)$ with Boyd indices verifying

$$
p<p_{X}=\sigma_{\varphi}^{a} \leq s_{\varphi}^{a}=q_{X}<q,
$$

we get that the function $\varphi$ is, up to equivalence, a strictly $p$-convex function and a strict $q$-convex function (see f.i. [Ma] pp. 22-24). Thus, the next proposition follows now from Theorem 3.A.

Proposition 10. Let $0<p<q<\infty$. If an Orlicz function space $L^{\varphi}(0, \infty)=X$ has Boyd indices $p<p_{X} \leq q_{X}<q$, then $L^{\varphi}(0, \infty)$ is order-isomorphic to a sublattice of $L^{p}(0, \infty)+L^{q}(0, \infty)$.

In the case $1 \leq p<q=\infty$, it also holds that the space $L^{p}(0, \infty)+$ $L^{\infty}(0, \infty)$ is universal for every Orlicz function space $L^{\varphi}(0, \infty)=X$ with Boyd indices $p<p_{X} \leq q_{X}<\infty$. This follows from the fact that the space $L^{p}(0, \infty)+L^{\infty}(0, \infty)$ contains an isomorphic copy of $l^{\infty}$ and the spaces $L^{\varphi}(0, \infty)$ are separable.

REMARK. In general the continuous inclusion map in Proposition 9, $L^{p}(0, \infty) \cap L^{q}(0, \infty) \subset X$, cannot be replaced by an isomorphic embedding as in the above proposition. For example, if $p=2<$ $r<q$, then the space $L^{2}(0, \infty) \cap L^{q}(0, \infty)$ which is isomorphic to $L^{q}(0, \infty)$ cannot be embedded into the space $L^{r}(0, \infty)$.

Finally, let us show that the last proposition does not hold in general for intermediate r.i. Banach spaces different from Orlicz function spaces:

EXAMPLE. Consider the Lorentz function space $X=L_{2,1}(0, \infty)$ defined as the space of all measurable functions $f$ on $(0, \infty)$ such that its decreasing rearrangement $f^{*}$ verifies

$$
\int_{0}^{\infty}\left(f^{*}(t)\right) t^{-1 / 2} d t<\infty
$$

It holds that its associated Boyd indices are $p_{X}=q_{X}=2$; hence by Proposition 9 , we have $L_{2,1}(0, \infty) \subset L^{p}(0, \infty)+L^{q}(0, \infty)$ for $1<p<2<q<\infty$.

However, the space $L_{2,1}(0, \infty)$ does not embed isomorphically into $L^{p}(0, \infty)+L^{q}(0, \infty)$. Indeed,

$$
L^{p}(0, \infty)+L^{q}(0, \infty)=L^{x^{p} \wedge x^{q}}(0, \infty)
$$


is a reflexive space, but the space $L_{2,1}(0, \infty)$ is not reflexive (it contains an $l^{1}$-complemented subspace, see [F-J-T]).

REMARK. We do not know whether there are Orlicz function spaces over the $[0,1]$-interval universal for intermediate classes of Orlicz function spaces $L^{\psi}[0,1]$, i.e. similar results to Theorems 3.A and 3.B for Orlicz spaces over finite measurable spaces $L^{\varphi}[0,1]$. Related to this question, it holds that the universal spaces $L^{p}(0, \infty)+L^{q}(0, \infty)$, for $1<p<q<2$, cannot be represented as a r.i. Banach function space on the $[0,1]$-interval (see [J-M-S-T] p. 230).

\section{REFERENCES}

[Bo] D. Boyd, Indices for Orlicz spaces, Pacific J. Math., 38 (1971), 315-323. [B-DC-K] J. Bretagnolle, D. Dacunha-Castelle, and J. L. Krivine, Lois stables et espaces $L^{p}$, Ann. Inst. Henri Poincaré, Sectión B, 2 (1966), 231-259.

[B-DC] J. Bretagnolle and D. Dacunha-Castelle, Application de l'etude de certaines formes linéaries aléatoires au plongement d'espaces de Banach dans des espaces $L^{p}$, Ann. Scient. Ec. Norm. Sup., $4^{e}$ série, 2 (1969), 437-480.

[D] S. J. Dilworth, A scale of linear spaces related to the $L_{p}$ scale, Illinois J. Math., 34 (1990), 140-158.

[F-J-T] T. Figiel, W. B. Johnson, and L. Tzafriri, On Banach lattices and spaces having local unconditional structures with applications to Lorentz function spaces, J. Approx. Theory, 13 (1975), 395-412.

[H-Ro] F. L. Hernández and B. Rodriguez-Salinas, On $l^{p}$-complemented copies in Orlicz spaces II, Israel J. Math., 68 (1989), 27-55.

[J-M-S-T] W. B. Johnson, B. Maurey, G. Schechtman, and L. Tzafriri, Symmetric structures in Banach spaces, Memoirs Amer. Math. Soc., no. 217, (1979).

[Lu] W. Luxemburg, Banach function spaces, Thesis Assen. (1955).

[L-T I ] J. Lindenstrauss and L. Tzafriri, Classical Banach Spaces I, SpringerVerlag, (1977).

[L-T II] _ Classical Banach Spaces II, Springer-Verlag, (1978).

[M] W. Matuszewska, Regularly increasing functions in connection with the theory of $L^{* \varphi}$-spaces, Studia Math., 21 (1962), 317-344.

[M-O] W. Matuszewska and W. Orlicz, A note on the theory of s-normed spaces of $\varphi$-integrable functions, Studia Math., 61 (1961), 107-115.

[Ma] L. Maligranda, Indices and interpolation, Dissertationes Math., 234 (1985).

[Mu J. Musielak, Orlicz spaces and modular spaces, Lect. Notes in Math., 1034 (1983).

[R] C. Ruiz, Estructura de Espacios de Orlicz de funciones y de sucesiones con pesos. Subespacios distinguidos, Doctoral Thesis, Universidad Complutense de Madrid, 1990.

[W] J. Y. T. Woo, On a class of universal modular sequence spaces, Israel J. Math., 20 (1975), 193-215.

Received May 18, 1990. Research supported in part by DGICYT PB88/0141. 


\section{PACIFIC JOURNAL OF MATHEMATICS EDITORS}

V. S. VARADARAJAN (Managing Editor)

University of California

Los Angeles, CA 90024-1555

vsv@math.ucla.edu

Herbert Clemens

University of Utah

Salt Lake City, UT 84112

clemens@math.utah.edu

F. Michael Christ

University of California

Los Angeles, CA 90024-1555

christ@math.ucla.edu

THOMAS ENRIGHT

University of California, San Diego

La Jolla, CA 92093

tenright@ucsd.edu
Nicholas ERcolani

University of Arizona

Tucson, AZ 85721

ercolani@math.arizona.edu

R. FINN

Stanford University

Stanford, CA 94305

finn@gauss.stanford.edu

VAUGHAN F. R. JoNES

University of California

Berkeley, CA 94720

vfr@math.berkeley.edu

STEVEN KeRCKHOFF

Stanford University

Stanford, CA 94305

spk@gauss.stanford.edu
C. C. MOORE

University of California

Berkeley, CA 94720

Martin ScharlemanN

University of California

Santa Barbara, CA 93106

mgscharl@henri.ucsb.edu

Harold Stark

University of California, San Diego

La Jolla, CA 92093

\begin{tabular}{|c|c|c|c|c|}
\hline \multicolumn{5}{|c|}{ ASSOCIATE EDITORS } \\
\hline R. ARENS & $\begin{array}{l}\text { E. F. BECKENBACH } \\
(1906-1982)\end{array}$ & B. H. NeumanN & $\begin{array}{c}\text { F. WoLF } \\
(1904-1989)\end{array}$ & K. YoshID \\
\hline \multicolumn{5}{|c|}{ SUPPORTING INSTITUTIONS } \\
\hline \multicolumn{2}{|c|}{ UNIVERSITY OF ARIZONA } & \multicolumn{3}{|c|}{ UNIVERSITY OF OREGON } \\
\hline \multicolumn{2}{|c|}{ UNIVERSITY OF BRITISH COLUMB } & \multicolumn{3}{|c|}{$\begin{array}{l}\text { UNIVERSITY OF SOUTHERN C } \\
\text { STANFORD UNIVERSITY }\end{array}$} \\
\hline \multicolumn{2}{|c|}{ CALIFORNIA INSTITUTE OF TECHNOLOGY } & \multirow{2}{*}{\multicolumn{3}{|c|}{$\begin{array}{l}\text { STANFORD UNIVERSITY } \\
\text { UNIVERSITY OF HAWAII }\end{array}$}} \\
\hline \multicolumn{2}{|c|}{ UNIVERSITY OF CALIFORNIA } & & & \\
\hline \multicolumn{2}{|c|}{ MONTANA STATE UNIVERSITY } & \multicolumn{3}{|c|}{ UNIVERSITY OF TOKYO } \\
\hline \multicolumn{2}{|c|}{ JNIVERSITY OF NEVADA, RENO } & \multicolumn{3}{|c|}{ UNIVERSITY OF UTAH } \\
\hline \multicolumn{2}{|c|}{ NEW MEXICO STATE UNIVERSITY } & \multicolumn{3}{|c|}{ WASHINGTON STATE UNIVERSITY } \\
\hline \multicolumn{2}{|c|}{ OREGON STATE UNIVERSITY } & \multicolumn{3}{|c|}{ UNIVERSITY OF WASHINGTON } \\
\hline
\end{tabular}




\section{PACIFIC JOURNAL OF MATHEMATICS}

Volume $155 \quad$ No. $1 \quad$ September 1992

Characterization of modular correspondences by geometric properties 1 Allan Russell AdLer

Representations of convex nondentable sets

SPIROS ARGYROS and IRENE DELIYANNI

Isomorphisms of spaces of continuous affine functions

CHO-Ho CHU and HENRY BRUCE COHEN

Universal classes of Orlicz function spaces

FRANCISCO LUIS HERNÁNDEZ RODRÍGUEZ and CESAR RUIZ

Asymptotic behavior of the curvature of the Bergman metric of the thin 99 domains

KANG-TAE KIM

Quadratic central polynomials with derivation and involution

CHARLES PHILIP LANSKI

Nonsplit ring spectra and products of $\beta$-elements in the stable homotopy of Moore spaces

\section{JIN KUN LIN}

Orientation and string structures on loop space

DENNIS MCLAUGHLIN

Homomorphisms of Bunce-Deddens algebras

\section{CORNEL PASNICU}

Certain $C^{*}$-algebras with real rank zero and their corona and multiplier 169 algebras. Part I

SHUANG ZHANG

Correction to: "On the density of twistor elementary states"

Michael G. Eastwood and A. M. Pilato 